\title{
Solution for Direct Kinematics of 3-PRS Parallel Manipulator Using Sylvester Dialytic Elimination Method
}

\author{
Bao-Lin YIN ${ }^{a}$, Bing-Bing YAN $^{b}$, Jun-Feng SHUAI ${ }^{c}$, Wen-Bo REN ${ }^{d}$ \\ Department of Mechanical Engineering, Jiamusi University, Jiamusi, China

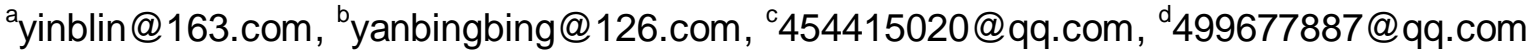

\begin{abstract}
Keywords: Parallel Manipulator, Direct Kinematics, Nonlinear System, Sylvester Dialytic
\end{abstract} Elimination.

\begin{abstract}
This paper presents the direct kinematics of a 3-PRS parallel manipulator. Through analysis position, the direct kinematics model is established, which is a nonlinear system of three equations in three unknown. The equations are derived using recursively the Sylvester dialytic elimination method. Finally, a numerical example is provided. Therefore, the direct kinematics is minimal..
\end{abstract}

\section{Introduction}

Parallel manipulator is a mechanism composed of a moving platform connected to a fixed base by means of at least two limbs [1]. Parallel manipulators have constituted a very active field of research over the last 20 years. Compared to serial manipulators, parallel manipulators essentially have two well-known advantages, namely greater precision in positioning and increased rigidity with respect to the relationship between size and workload limit. Many parallel manipulators with less than six Degrees Of Freedom (DOF) have been introduced, such as the famous DELTA robot with three translational DOF [2].

One of the challenges in studying parallel manipulators consists of the difficulty in solving their direct kinematics problems, which leads to systems of polynomial equations [3]. For direct kinematics, the input joint variables are given and all possible moving platform positions that would result from the given input values need to be found. Direct kinematic analysis is an essential component of the design, programming and control of any mechanism. Solution approaches for such a problem can be divided into two classes: numerical methods and analytic techniques [4]. To find all possible solutions of the direct kinematics problems of parallel manipulators, analytical techniques are found in the literature $[5,6]$. Three common approaches to solving these systems of polynomial equations are the Dialytic Elimination, Polynomial Continuation and Grobner bases.

The purpose of this paper is to solve the direct kinematics problem of a 3-PRS parallel manipulator. Deriving the equations governing the problem leads to three couple equations. Then the equations are derived using recursively the Sylvester dialytic elimination method. Finally, a numerical example is provided.

\section{Description of the 3-PRS Parallel Manipulator}

The schematic of the 3-PRS parallel manipulator is shown in Fig. 1. The architecture of the manipulator is composed of a moving platform, a fixed base and three PRS type active limbs with the linear actuators fixed the base. Each PRS type active limb connects the moving platform to the base by a prismatic joint, $\mathrm{P}$ at $B_{i}$, a revolute joint at $C_{i}$, and a spherical joint at $M_{i}$. The prismatic joint is actuated and the other joints are passive. A reference frame $O-x y z$ is attached to the base at point $O$, located at the center of the base. The $z$ axes is perpendicular to the base. Point $B_{i}$ is assumed to lie at a radial distance of $r_{\mathrm{B}}$ from the point $O . B_{i} C_{i}$ is the direction of the prismatic joint movement, which is parallel to $z$ axes. The axes of the revolute joint is perpendicular to the $B_{i} O$ and $B_{i} C_{i}$. Point $O_{\mathrm{M}}$ is the center of the moving platform. And point $M_{i}$ is the center of the spherical joint, which is assumed to lie at a radial distance of $r_{\mathrm{M}}$ from the point $O_{\mathrm{M}}$. The distance from $C_{i}$ to $M_{i}$ is $L$. The angle $\varphi_{i}$ is defined from $B_{i} C_{i}$ to $C_{i} M_{i}$. And the angle $\theta_{\mathrm{B} i}$ is defined from $x$ axes to $O B_{i}$. The drive parameters $q_{i}$ is the distance from $B_{i}$ to $C_{i}$. 


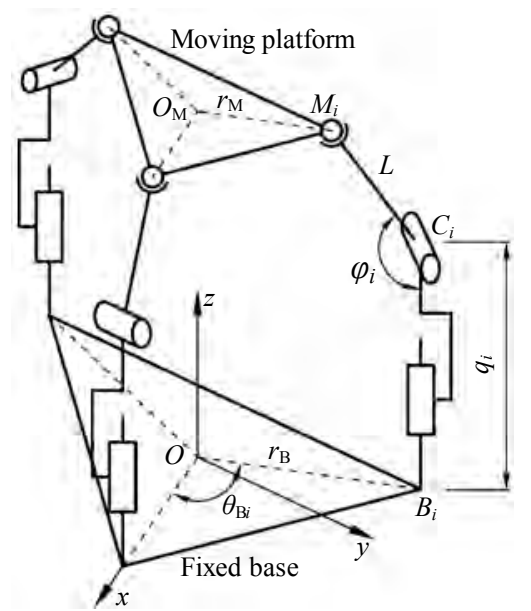

Fig. 1 Schematic of the 3-PRS parallel manipulator

\section{Direct Kinematics}

The position of $M_{i}$ in the $O-x y z$ coordinate frame for the $i^{t h}$ limb can be expressed as $x_{\mathrm{M} i}, y_{\mathrm{M} i}$ and $z_{\mathrm{M} i}$ $\left\{\begin{array}{l}x_{\mathrm{M} i}=\left(r_{\mathrm{B}}-L \sin \varphi_{i}\right) \cos \theta_{\mathrm{B} i} \\ y_{\mathrm{M} i}=\left(r_{\mathrm{B}}-L \sin \varphi_{i}\right) \sin \theta_{\mathrm{B} i} \quad(i=1 \sim 3) . \\ z_{\mathrm{M} i}=q_{i}-L \cos \varphi_{i}\end{array}\right.$

As the distance between the $M_{i}$ are known constant, and it can be expressed as

$$
\begin{aligned}
& f_{12}:\left\|M_{1}-M_{2}\right\|=d_{12} \\
& f_{13}:\left\|M_{1}-M_{3}\right\|=d_{13} \\
& f_{23}:\left\|M_{2}-M_{3}\right\|=d_{23} .
\end{aligned}
$$

where $d_{i j}$ is the distance between point $M_{i}$ to $M_{j}$. Substituting Eq. 1 into Eq. 2, yields

$$
f_{i j}: u_{i j 1} \cos \varphi_{i} \cos \varphi_{i}+u_{i j 2}\left(\cos \varphi_{i}-\cos \varphi_{j}\right)+u_{i j 3} \sin \varphi_{i} \sin \varphi_{i}+u_{i j 4}\left(\sin \varphi_{i}+\sin \varphi_{j}\right)+u_{i j 5}=0
$$

where $u_{i j k}$ can be expressed as

$$
\begin{aligned}
& u_{i j 1}=-2 L^{2} \\
& u_{i j 2}=-2 L\left(q_{i}-q_{j}\right) \\
& u_{i j 3}=-2 L^{2}\left(\cos \theta_{\mathrm{B} i} \cos \theta_{\mathrm{B} j}+\sin \theta_{\mathrm{B} i} \sin \theta_{\mathrm{B} j}\right) \\
& u_{i j 4}=2 L r_{\mathrm{B}}\left(\cos \theta_{\mathrm{B} i} \cos \theta_{\mathrm{B} j}+\sin \theta_{\mathrm{B} i} \sin \theta_{\mathrm{B} j}-1\right) \\
& u_{i j 5}=-2 r_{\mathrm{B}}^{2}\left(\cos \theta_{\mathrm{B} i} \cos \theta_{\mathrm{B} j}+\sin \theta_{\mathrm{B} i} \sin \theta_{\mathrm{B} j}-1\right)+\left(q_{i}-q_{j}\right)^{2}+2 L^{2}-d_{i j}^{2} .
\end{aligned}
$$

The half-angel tangent relationships can be expressed as

$$
\sin \varphi_{i}=\frac{2 t_{i}}{1+t_{i}^{2}} \text { and } \cos \varphi_{i}=\frac{1-t_{i}^{2}}{1+t_{i}^{2}} .
$$

Substituting Eq. 5 into Eq. 4, direct kinematics of the 3-PRS parallel manipulator can be shown as

$$
F_{i j}: t_{i}^{2} t_{j}^{2}+v_{i j 1}\left(t_{i}^{2} t_{j}+t_{i} t_{j}^{2}+t_{i}+t_{j}\right)+v_{i j 2} t_{i}^{2}+v_{i j 3} t_{j}^{2}+v_{i j 4} t_{i} t_{j}+1=0 \text {. }
$$

where $v_{i j k}$ can be expressed as 


$$
\begin{aligned}
& v_{i j 1}=2 u_{i j 4} /\left(u_{i j 1}+u_{i j 5}\right) \\
& v_{i j 2}=\left(-u_{i j 1}-2 u_{i j 2}+u_{i j 5}\right) /\left(u_{i j 1}+u_{i j 5}\right) \\
& v_{i j 3}=\left(-u_{i j 1}+2 u_{i j 2}+u_{i j 5}\right) /\left(u_{i j 1}+u_{i j 5}\right) \\
& v_{i j 4}=4 u_{i j 3} /\left(u_{i j 1}+u_{i j 5}\right)
\end{aligned}
$$

Eq. 6 is a nonlinear system of three equation in three unknown $t_{1}, t_{2}$ and $t_{3}$. The Dialytic elimination method is applied to $F_{23}$ and $F_{13}$, producing the following equation

$$
\left[\begin{array}{cccc}
t_{1}^{2}+v_{13,1} t_{1}+v_{13,3} & v_{13,1} t_{1}^{2}+v_{13,4} t_{1}+v_{13,1} & v_{13,2} t_{1}^{2}+v_{13,1} t_{1}+1 & 0 \\
0 & t_{1}^{2}+v_{13,1} t_{1}+v_{13,3} & v_{13,1} t_{1}^{2}+v_{13,4} t_{1}+v_{13,1} & v_{13,2} t_{1}^{2}+v_{13,1} t_{1}+1 \\
t_{2}^{2}+v_{23,1} t_{2}+v_{23,3} & v_{23,1} t_{2}^{2}+v_{23,4} t_{2}+v_{23,1} & v_{23,2} t_{2}^{2}+v_{23,1} t_{2}+1 & 0 \\
0 & t_{2}^{2}+v_{23,1} t_{2}+v_{23,3} & v_{23,1} t_{2}^{2}+v_{23,4} t_{2}+v_{23,1} & v_{23,2} t_{2}^{2}+v_{23,1} t_{2}+1
\end{array}\right]\left[\begin{array}{c}
t_{3}^{3} \\
t_{3}^{2} \\
t_{3} \\
1
\end{array}\right]=0
$$

For a nontrivial solution exist for Eq.8, the determinant of the square matrix must equal 0 . This is developed as

$$
F_{(13)(23)}: n_{1} t_{2}^{4}+n_{2} t_{2}^{3}+n_{3} t_{2}^{2}+n_{4} t_{2}+n_{5}=0 .
$$

where $n_{i}$ can be expressed as

$$
\begin{aligned}
& n_{1}=o_{11} t_{1}^{4}+o_{12} t_{1}^{3}+o_{13} t_{1}^{2}+o_{14} t_{1}+o_{15} \\
& n_{2}=o_{21} t_{1}^{4}+o_{22} t_{1}^{3}+o_{23} t_{1}^{2}+o_{24} t_{1}+o_{25} \\
& n_{3}=o_{31} t_{1}^{4}+o_{32} t_{1}^{3}+o_{33} t_{1}^{2}+o_{34} t_{1}+o_{35} \\
& n_{4}=o_{41} t_{1}^{4}+o_{42} t_{1}^{3}+o_{43} t_{1}^{2}+o_{44} t_{1}+o_{45} \\
& n_{5}=o_{51} t_{1}^{4}+o_{52} t_{1}^{3}+o_{53} t_{1}^{2}+o_{54} t_{1}+o_{55} .
\end{aligned}
$$

$F_{12}$ is developed as

$$
F_{12}: m_{1} t_{2}^{2}+m_{2} t_{2}+m_{3}=0 \text {. }
$$

where $m_{i}$ can be expressed as

$$
\begin{aligned}
& m_{1}=t_{1}^{2}+v_{12,1} t_{1}+v_{12,3} \\
& m_{2}=v_{12,1} t_{1}^{2}+v_{12,4} t_{1}+v_{12,1} \\
& m_{3}=v_{11,2} t_{1}^{2}+v_{11,1} t_{1}+1
\end{aligned}
$$

The Dialytic elimination method is applied to $F_{(13)(23)}$ and $F_{12}$, producing the following equation

$$
\left[\begin{array}{cccccc}
m_{1} & m_{2} & m_{3} & 0 & 0 & 0 \\
0 & m_{1} & m_{2} & m_{3} & 0 & 0 \\
0 & 0 & m_{1} & m_{2} & m_{3} & 0 \\
0 & 0 & 0 & m_{1} & m_{2} & m_{3} \\
n_{1} & n_{2} & n_{3} & n_{4} & n_{5} & 0 \\
0 & n_{1} & n_{2} & n_{3} & n_{4} & n_{5}
\end{array}\right]\left[\begin{array}{c}
t_{2}^{5} \\
t_{2}^{4} \\
t_{2}^{3} \\
t_{2}^{2} \\
t_{2} \\
1
\end{array}\right]=0
$$

Eq. 13 produces a $16^{\text {nd }}$ degree polynomial in $t_{1}$. This equation can be solved for $t_{1}$, and then the values of $t_{2}$ and $t_{3}$ are determined by back-substitution. 


\section{Numerical Example}

As an example of the direct kinematics solution for a general case mechanism, let the parameters be as: $r_{\mathrm{B}}=0.30, \theta_{\mathrm{B} 1}=0, \theta_{\mathrm{B} 2}=2 \pi / 3, \theta_{\mathrm{B} 3}=4 \pi / 3, r_{\mathrm{M}}=0.18, d_{i j}=\sqrt{3} r_{\mathrm{M}}, L=0.22, q_{1}=0.16, q_{1}=0.22, q_{3}=0.26$. The roots for $t_{i}$ are given in Table 1 . There are 16 solutions, and in which there are 8 real roots. So, for the given drive parameters $q_{i}$, there are 8 possible poses for the 3 -PRS parallel mechanism. Those real roots, which are depicted in Fig. 2, correspond to the assembly modes of the manipulator. Therefore, the direct kinematics is minimal.

Tab.1 Solutions of $t_{i}$ for the Direct Kinematics

\begin{tabular}{|c|c|c|c|c|c|c|c|}
\hline No. & $t_{1}$ & $t_{2}$ & $t_{3}$ & No. & $t_{1}$ & $t_{2}$ & $t_{3}$ \\
\hline 1 & 0.3552134 & 0.2521578 & 0.8071060 & 9 & $\begin{array}{c}-1.1643614 \\
-0.4539659 \mathrm{I}\end{array}$ & $\begin{array}{c}0.4648174 \\
+0.9031792 \mathrm{I}\end{array}$ & $\begin{array}{c}0.4506974 \\
+0.8752161 \mathrm{I}\end{array}$ \\
\hline 2 & 0.3257314 & 0.2829163 & 0.3251804 & 10 & $\begin{array}{c}0.4679279 \\
+0.9105487 \mathrm{I}\end{array}$ & $\begin{array}{c}0.4462254 \\
+0.8689908 \mathrm{I}\end{array}$ & $\begin{array}{c}-0.7681163 \\
-0.2933693 \mathrm{I}\end{array}$ \\
\hline 3 & 1.5397644 & 0.3319772 & 0.2676018 & 11 & $\begin{array}{c}0.4697463 \\
+0.8870099 \mathrm{I}\end{array}$ & $\begin{array}{c}0.4542512 \\
+0.8910920 \mathrm{I}\end{array}$ & $\begin{array}{c}0.4638804 \\
+0.8834293 \mathrm{I}\end{array}$ \\
\hline 4 & 4.3773386 & 0.8363213 & 2.4761929 & 12 & $\begin{array}{c}0.4724809 \\
-0.9259680 \mathrm{I}\end{array}$ & $\begin{array}{c}-0.9157076 \\
+0.3263262 \mathrm{I}\end{array}$ & $\begin{array}{c}0.4371669 \\
-0.8569080 \mathrm{I}\end{array}$ \\
\hline 5 & 0.4047012 & 1.1001490 & 0.2276883 & 13 & $\begin{array}{c}0.4724809 \\
+0.9259680 \mathrm{I}\end{array}$ & $\begin{array}{c}-0.9157076 \\
-0.3263262 \mathrm{I}\end{array}$ & $\begin{array}{c}0.4371669 \\
+0.8569080 \mathrm{I}\end{array}$ \\
\hline 6 & 3.9293282 & 2.8349249 & 0.6712913 & 14 & $\begin{array}{c}0.4697463 \\
-0.8870099 \mathrm{I}\end{array}$ & $\begin{array}{c}0.4542512 \\
-0.8910920 \mathrm{I}\end{array}$ & $\begin{array}{c}0.4638804 \\
-0.8834293 \mathrm{I}\end{array}$ \\
\hline 7 & 2.9185724 & 3.5289475 & 3.1583887 & 15 & $\begin{array}{c}0.4679279 \\
-0.9105487 \mathrm{I}\end{array}$ & $\begin{array}{c}0.4462254 \\
-0.86899908 \mathrm{I}\end{array}$ & $\begin{array}{c}-0.7681163 \\
+0.2933693 \mathrm{I}\end{array}$ \\
\hline 8 & 1.3284484 & 1.3284484 & 2.9984559 & 16 & $\begin{array}{c}-1.1643614 \\
+0.4539659 \mathrm{I}\end{array}$ & $\begin{array}{c}0.4648174 \\
-0.9031792 \mathrm{I}\end{array}$ & $\begin{array}{c}0.4506974 \\
-0.8752161 \mathrm{I}\end{array}$ \\
\hline
\end{tabular}

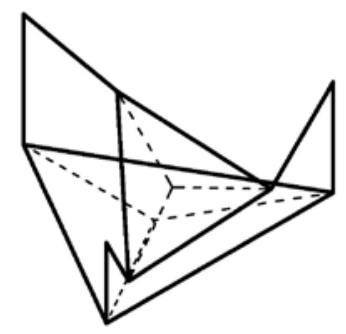

No. 1

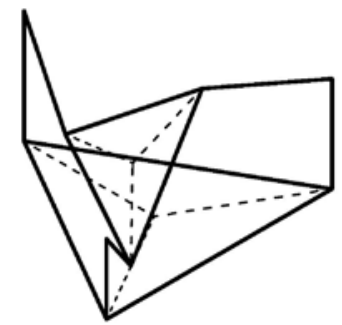

No. 5

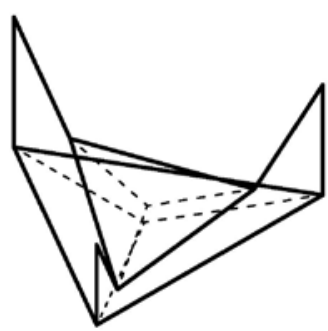

No. 2

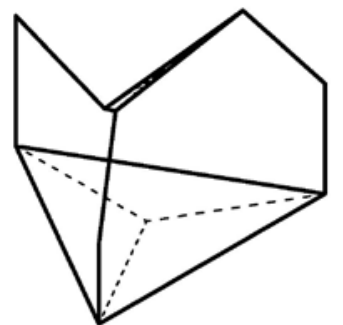

No. 6

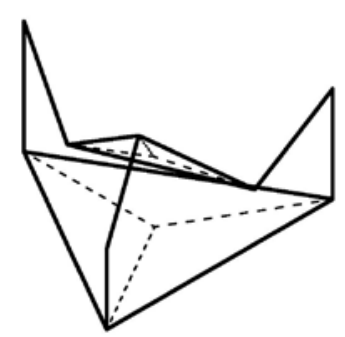

No. 3

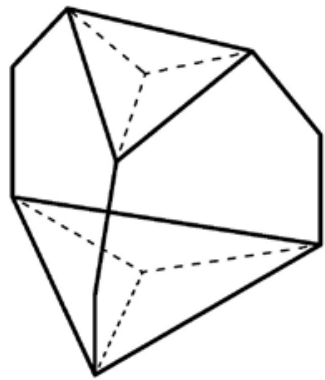

No. 7

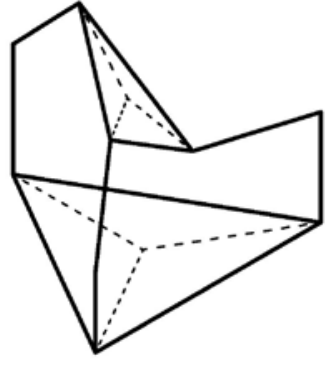

No. 4

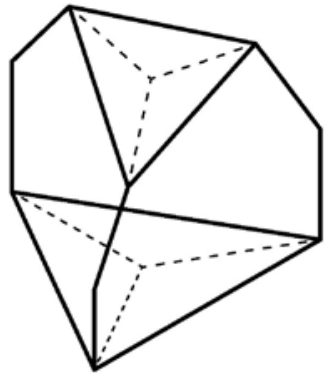

No. 8

Fig. 2 The Eight Assembly Model of the Example

\section{Conclusion}

In this paper, the direct kinematics of 3-PRS parallel manipulation has been successfully approached. Firstly, the position analysis was carried out, and direct kinematics of the 3-PRS parallel manipulator was established. The direct kinematics, which was nonlinear system, was carried out using Sylvester dialytic elimination method. Then, a $16^{\text {nd }}$ degree polynomial in one unknown was produced. The results imply 
that, for a set of three given drive parameters, the foregoing polynomial admitted up to sixteen solutions. A numerical example having eight real solutions was included. Therefore, the polynomial is minimal.

\section{Acknowledgement}

This work has been supported by science and technology research projects of Heilongjiang Province Office of Education (Item Number 12531671).

\section{References}

[1]D. Stewart: Proceedings of the Institution of Mechanical Engineer, Vol. 180, p. 371-386 (1965).

[2]R. Clavel: Proceeding of the International Symposium on I ndustrial Robots, Lausanne, p. 91-100(1988).

[3]J. P. Merlet: IEEE Transactions on Robotics and Automation, Vol. 6, p. 842-846 (1993).

[4]H. Sadjadian and H.D. Taghirad: Journal of Intelligent and Robotic Systems, Vol. 44, p. 225-246 (2005).

[5]G. Abbasnejad, H.M. Daniali and A. Fathi: Scientia Iranica B, Vol. 2, p. 320-326 (2012).

[6]J. Gallardo, H. Orozco and J.M. Rico: Int. J. Adv. Manuf. Technol. Vol. 36, p. 598-605 (2008). 